2020, Vol: 21, pp. 40-44, DOI: 10.18038/estubtda.818452

\title{
A COMPARATIVE STUDY OF CLASSIFICATION METHODS ON HUMAN SKIN DETECTION FROM RGB AND YCBCR REPRESENTED COLOR IMAGES
}

\author{
Utku KAYA ${ }^{1, *(D)}$, Murat BAŞARAN ${ }^{2}$ \\ ${ }^{1}$ Vocational School of Transportation, Eskișehir Technical University Eskișehir, Turkey \\ ${ }^{2}$ Vocational School of Transportation, Eskişehir Technical University Eskişehir, Turkey
}

\begin{abstract}
Skin detection has an important place in image processing. Human-computer interaction has made this study area very popular. The most common color space used in skin detection is Red Green and Blue but RGB space can be converted into YCbCr space. Both features give strong information about the properties of the images. In this study, RGB and YCbCr spaces are used to detect human skin. The extracted features are trained by several classification methods. The obtained features are used to segment the human skin by using the chosen classification algorithm and finally, the promising performance results are presented comparatively with the most commonly used classifications methods in the literature.
\end{abstract}

Keywords: Feature extraction, Image segmentation, $\mathrm{YCbCr}$

\section{INTRODUCTION}

Skin detection is an important application of image processing aimed at identifying pixels related to human skin in color images. Skin detection is a technique that is constantly being developed and widely used. The enormous increase in the use of mobile devices, face detection, and recognition techniques have played an important role in secure and accurate user authentication on devices.

Skin detection is a difficult process. Because the pixels representing the skin on the images are affected by external factors such as ambient light, daylight, bright light illumination. Such effects have a filter effect on the images and affect the detection accuracy. Besides, blurring caused by the movement of the person and the lens characteristics of the different types of cameras used to make skin detection difficult. Besides the external effects mentioned above, the fact that the surrounding objects have a color close to the human skin, the different ethnic origin, race, age, gender, having different colors of different body parts of the people, the use of makeup, hairstyle and glasses affect skin detection success significantly.

In human and computer interaction, there has been a transition to hand and face tracking and gesture analysis instead of mechanical systems. Skin detection is therefore very important. Also, skin detection can determine the detection of various skin diseases better than the human eye [1]. It is also used to filter adult images on websites [2].

\section{EXPERIMENTAL METHOD}

\subsection{Feature Extraction for Skin Segmentation}

The most common uses in the definition of colors are RGB color band (red, green, blue). Besides, the graphics software offers the YCbCr band (hue, saturation, value) in the color picker [3], [4]. In this color space, $\mathrm{Y}$ denotes the luminescent component of the color. The luminescent component refers to the light intensity of the color and is a component that the human eye is sensitive to. There is a proportional relationship between luminescent and brightness. $\mathrm{Cb}$ and $\mathrm{Cr}$ are color components representing the relationship of blue component and red components with the green component, respectively. The human

*Corresponding Author: utkukaya@eskisehir.edu.tr

Received: 30.10.2020 Published:27.11.2020 
eye is less sensitive to these components than the luminescent component. Since the Y component is more sensitive to the human eye, the error tolerance is lower. Conversely, the error tolerance is higher as the human eye is less sensitive to the $\mathrm{Cb}$ and $\mathrm{Cr}$ components. Therefore, when JPEG standard pictures are compressed, they use these sensitivities of the human eye, and the undetectable details of the colors in the picture are eliminated. $\mathrm{Y}, \mathrm{Cb}$ and $\mathrm{Cr}$ calculations are given in Equations (1), (2) and (3).

$$
\begin{aligned}
& Y=16+(65.481 \times \hat{R}+128.553 \times \hat{G}+24.966 \times \hat{B}) \\
& C b=128+\left(\begin{array}{ll}
37.797 \times \hat{R} & 74.203 \times \hat{G}+112.0 \times \hat{B}
\end{array}\right) \\
& C r=128+\left(\begin{array}{lll}
112.0 \times \hat{R} & 93.786 \times \hat{G} & 18.214 \times \hat{B}
\end{array}\right)
\end{aligned}
$$

All the data used was taken from the image dataset specified in Schumugge's article [5]. This dataset consisting of 845 images (consisting of more than 18.6 million pixels) with manual ground truth. The images were taken in different environments (indoor and outdoor) within the dataset include people of different races and origins. There are a lot of photos in this data set that are affected by the ambient conditions mentioned earlier. $\mathrm{YCbCr}$ values of these photographs were calculated with equations 1,2 and 3. Six features have been created, including RGB and $\mathrm{YCbCr}$ as seen in table 1. Each of the 6 features has labels, corresponding skin, and nonskin.

Table 1. Proposed features for skin segmentation

\begin{tabular}{cc}
\hline Feature No & Feature Description \\
\hline 1 & Red \\
\hline 2 & Green \\
3 & Blue \\
4 & Luminance $(\mathrm{Y})$ \\
5 & Blue Difference $(\mathrm{Cb})$ \\
6 & Red Difference $(\mathrm{Cr})$ \\
\hline
\end{tabular}

\subsection{Training}

The high number of pixels corresponding to the nonskin negatively affects the classification performance. This is called unbalanced classification. This is stated in the following articles [6], [7], [8]. Therefore, the nonskin pixel count has been eliminated to equal the number of pixels skin. The obtained pixels were trained with MATLAB. Table 2 shows the classification methods and their success.

Table 2. Accuracy performances of all selected classifiers

\begin{tabular}{ll}
\hline Classification method & Accuracy \\
\hline Tree (Fine Tree) & $\% 87.4$ \\
Tree (Medium Tree) & $\% 87.0$ \\
Tree (Coarse Tree) & $\% 84.8$ \\
Linear Discriminant & $\% 84.8$ \\
Logistic Regression & $\% 85.2$ \\
Naive Bayes (Gaussian) & $\% 81.5$ \\
Naive Bayes (Kernel) & $\% 81.4$ \\
SVM (Linear SVM) & $\% 84.9$ \\
SVM (Fine Gaussian SVM) & $\% 88.2$ \\
SVM (Medium Gaussian SVM) & $\% 87.5$ \\
SVM (Coarse Gaussian SVM) & $\% 85.8$ \\
KNN (Fine KNN) & $\% 84.5$ \\
KNN (Medium KNN) & $\% 88.0$ \\
KNN (Coarse KNN) & $\% 87.4$ \\
KNN (Cosine KNN) & $\% 86.5$ \\
KNN (Weighted KNN) & $\% 87.2$ \\
Ensemble (Boosted Trees) & $\% 87.5$ \\
Ensemble (Bagged Trees) & $\% 87.8$ \\
\hline
\end{tabular}


The accuracy values shown in Table 2 are the success of the test data in the data is used. When a trained model is tested with other images, it will show a different success. Although our goal is to detect skin pixels, nonskin detection and elimination are also important.

The success of the classifier only in the skin pixel (positive class) is measured by the sensitivity parameter and the sensitivity parameter is calculated by Equation (4).

$$
\text { Sensitivity }=\frac{T P}{P}
$$

The success of the classifier only in non-skin pixel (negative class) is measured by the specificity parameter and the Specificity parameter is calculated by Equation (5).

$$
\text { Specificity }=\frac{\mathrm{TN}}{\mathrm{N}}
$$

Balanced accuracy is obtained from the arithmetic mean of the Sensitivity and Specificity parameters, as shown in Equation (6).

$$
\text { Balanced Accuracy }=\frac{\text { Sensitivity }+ \text { Specificity }}{2}
$$

Since features are trained by equalizing the number of skin and nonskin pixels, PPV, NPV, F1 scores were not calculated. The most successful methods among the classification methods that seem in Table 2 SVM (Fine Gaussian), KNN (Medium KNN) were selected and the least successful method Naive Bayes were used to showing difference The model was tested with the test pictures in Figure 1 and 2 and the successes is calculated.

\section{RESULTS}

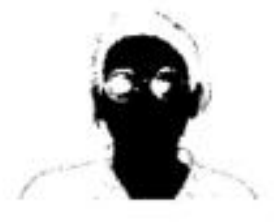

1.Naive Bayes (Kernel)

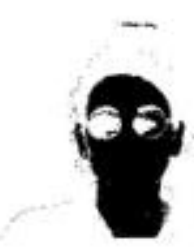

3.KNN (Medium KNN)

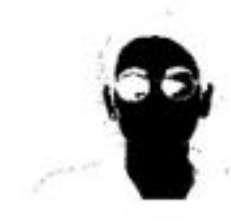

2.SVM (Fine Gaussian)

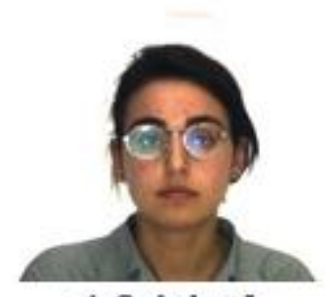

4.Original

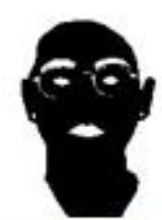

5.Labeled

Figure 1. The selected image (Face oriented, white background)

Figure 1 shows that all classification methods correctly detect skin pixels. The success rates are expressed as arithmetic in Table 3 to understand which method is more successful. 
Table 3. Classification performances of images in figure. 1

\begin{tabular}{|c|c|c|c|}
\hline & Sensitivity & Specificity & Balanced_accuracy \\
\hline $\begin{array}{c}\text { Naive Bayes } \\
\text { (Kernel) }\end{array}$ & 0.8942 & 0.9816 & 0.9379 \\
\hline $\begin{array}{c}\text { SVM (Fine } \\
\text { Gaussian) }\end{array}$ & 0.8369 & 0.9916 & 0.9142 \\
\hline $\begin{array}{c}\text { KNN (Medium } \\
\text { KNN) }\end{array}$ & 0.8400 & 0.9890 & 0.9145 \\
\hline
\end{tabular}

Naive Bayes, who was expected to show low success, achieved successful skin detection compared to other classification methods (sensitivity $=0.8942$ ), but the SVM (fine gaussian) is most successful in detecting non-skin pixels (specificity $=0.9916$ ). When looking at the balance accuracy values, it is seen that the most successful method is Naive Bayes.

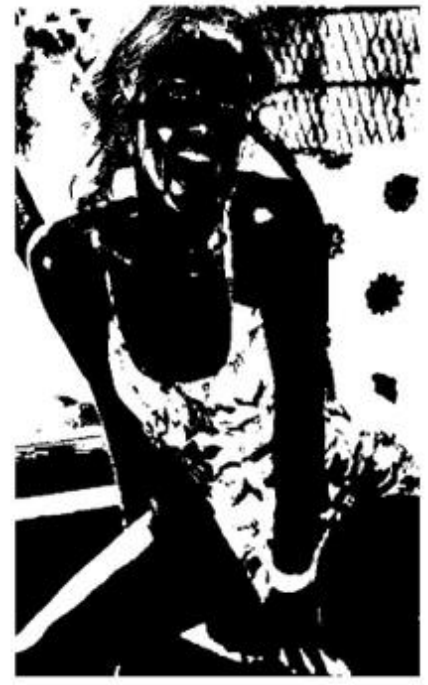

1.Naive Bayes (Kernel)

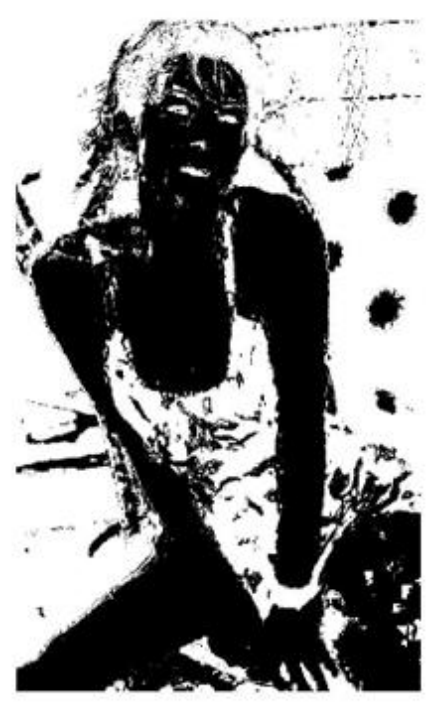

3.KNN (Medium KNN)

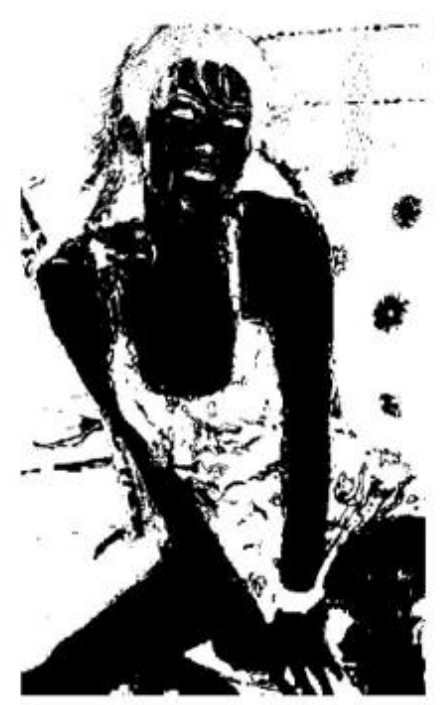

2.SVM (Fine Gaussian)

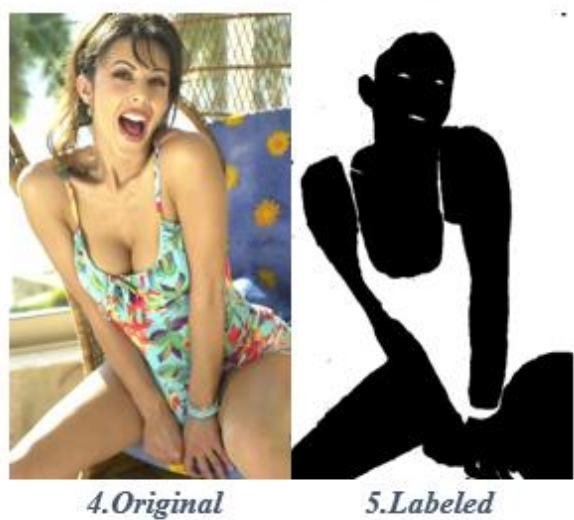

Figure 2. The selected image (Face and body, colorful background) 
Table 4. Classification performances of the images in figure 2

\begin{tabular}{|c|c|c|c|}
\hline $\begin{array}{c}\text { Naive Bayes } \\
\text { (Kernel) }\end{array}$ & Sensitivity & Specificity & Balanced_accuracy \\
\hline $\begin{array}{c}\text { SVM (Fine } \\
\text { Gaussian) }\end{array}$ & 0.9182 & 0.6061 & 0.7621 \\
\hline $\begin{array}{c}\text { KNN (Medium } \\
\text { KNN) }\end{array}$ & 0.8614 & 0.8719 & 0.8625 \\
\hline
\end{tabular}

In order to show the effects of different classification methods on different images, a different image containing the face and body with a high number of skin pixels was used and tested with the classification methods that are mentioned. A striking point in this photo is that the background is not white.

In the image that is used in figure 1, while Naive Bayes was very successful, in figure 2 failed (specificity $=0.6061$ ). Although Sensitivity (skin detection success) is seen in the highest with Naive Bayes, the value of Specificity (non-skin success) is extremely low compared to other classifiers. The failure of the Naive Bayes method to detect non-skin objects can be seen in figure 2. When looking at the balanced accuracy values for this image, the method with the highest success is the support vector machine (SVM) classifier.

\section{CONCLUSIONS}

This study aimed to separate skin pixels from images by using RGB and YCbCr. The successes of different classification methods in different types of images were tested and information was given about the successes of each classification method. Two different classifiers were found to be successful in two different photos given. While the success of the Naive Bayes method was noticeable in the first photo that is used, it was observed that in the 2nd photo, SVM method was the most successful.

\section{REFERENCES}

[1] Sumithra R, Mahamad Suhil and D S. Guru. Segmentation and Classification of Skin Lesions for Disease Diagnosis. Procedia Computer Science 45 (2015): 76-85.

[2] Lee Jiann-Shu, et al. Naked Image Detection Based on Adaptive and Extensible Skin Color Model. Pattern Recognition 40.8 (2007): 2261-2270.

[3] Basilio Jorge Alberto Marcial, et al. Explicit Image Detection using YCbCr Space Color Model as Skin Detection Applications of Mathematics and Computer Engineering (2011): 123-128.

[4] Shaik Khamar Basha, et al. Comparative Study of Skin Color Detection and Segmentation in HSV and YCbCr Color Space. Procedia Computer Science 57.12 (2015): 41-48.

[5] Schmugge SJ, et al. Objective Evaluation of Approaches of Skin Detection using ROC Analysis. Computer Vision and Image Understanding 108.1-2 (2007): 41-51.

[6] Dal Pozzolo Andrea et al. Calibrating Probability with Undersampling for Unbalanced Classification. 2015 IEEE Symposium Series on Computational Intelligence. IEEE, 2015.

[7] Dal Pozzolo, Andrea, Olivier Caelen, and Gianluca Bontempi. When is undersampling effective in unbalanced classification tasks? Joint European Conference on Machine Learning and Knowledge Discovery in Databases. Springer, Cham, 2015.

[8] Grandvalet, Yves, Johnny Mariéthoz, and Samy Bengio. A Probabilistic Interpretation of SVMs with An Application to Unbalanced Classification. Advances in Neural Information Processing Systems. 2006. 